\title{
Ownership, utilization, and motivations for use or non-use of bed nets among community members at risk of malaria along the Thai-Myanmar border
}

\section{Kasama Pooseesod}

Mahidol University Faculty of Tropical Medicine

Daniel M. Parker

University of California Irvine Department of Population Health \&amp; Disease Prevention

Natthani Meemon

Mahidol University Faculty of Social Science and Humanities

Saranath Lawpoolsri

Mahidol University Faculty of Tropical Medicine

Pratap Singhasivanon

Mahidol University Faculty of Tropical Medicine

Jetsumon Sattabongkot

Mahidol University Faculty of Tropical Medicine

Liwang Cui

University of South Florida College of Medicine: USF Health Morsani College of Medicine

SUPARAT PHUANUKOONNON ( $\nabla$ suparat.phu@mahidol.ac.th )

Mahidol University Faculty of Tropical Medicine https://orcid.org/0000-0003-4670-4006

\section{Research Article}

Keywords: Malaria, bed net, Long-lasting insecticide net, Karen ethnic, Forest goers, Thailand

Posted Date: April 14th, 2021

DOI: https://doi.org/10.21203/rs.3.rs-418488/v1

License: (c) (1) This work is licensed under a Creative Commons Attribution 4.0 International License.

Read Full License

Version of Record: A version of this preprint was published at Malaria Journal on July 6th, 2021. See the published version at https://doi.org/10.1186/s12936-021-03837-5. 


\section{Abstract \\ Background}

With the goal for malaria elimination in Thailand set for 2024, increased coverage and utilization of bed net, especially insecticide-treated net (ITN) or long-lasting insecticidal net (LLIN) is a key strategy. This study aims to provide the necessary information about bed net ownership and utilization among the population at risk of malaria living along the Thai-Myanmar border in Tak province.

\section{Methods}

A cross-sectional study was conducted using a mixed-method approach in 331 households from 5 hamlets in the villages of the Thai-Myanmar border. The research tools included a questionnaire, bed net inspection, and semi-structured interviews. Log-binomial regression was used to explore the sociodemographic factors associated with bed net utilization. The qualitative analysis employed a thematic analysis approach.

\section{Results}

This survey found that $98.5 \%$ of households had at least one bed net per household, and $74.3 \%$ had at least one ITN/LLIN. However, only $30.8 \%$ of households reached the standard policy set by the Minister of Public Health of one ITN/LLINs per two persons. Most residents used bed net ( $92.1 \%$ used in the previous night and $80.9 \%$ used every day). For those using bed nets, however, $61.9 \%$ used ITNs or LLINs the night before and $53.1 \%$ used them every day. Nonetheless, the usage rates of bed nets (any type) in the previous night among children and pregnant women were high, reaching $95.3 \%$ and $90.0 \%$, respectively. Three explanatory variables including "not stay overnight in the forest", "preference of free bed nets", and "sufficient numbers of bed nets to cover all sleeping spaces", showed statistically significant association with bed net use every day. The major reasons for the regular use of bed nets in both household and the forest were to prevent mosquito biting. The reasons for not using bednets in the household were discomfort feelings from heat, perception of unnecessity due to low mosquito density, whereas the reason for not using bednets in the forest was inconvenience.

\section{Conclusion}

Despite that overall coverage and usage of bed nets was high, only one third reached the standard level specified by the policy. Overnight in the forest, the dissatisfaction with the quality of free bed nets, insufficient number of bed nets, discomfort from heat, perception of no benefits of bed nets due to low mosquito density, and inconvenience were factors influencing bed net use. Maintaining high coverage and utility rate of bed nets should be a priority for the malaria high-risk population. 


\section{Background}

Based on the 2020 world malaria report, there were 87 countries and areas with ongoing malaria transmission and approximately 229 million malaria cases in 2019 [1].Thailand reported a substantial decline (19\%) in total reported cases between 2018 and 2019 [2]. Malaria in Thailand is patchy in its distribution and can be typified as 'border malaria' and 'forest malaria', with the highest transmission along international borders and in rural forested areas [3]. The western border with Myanmar has had the highest burden of malaria and has been the focus of malaria control programs for decades [3]. Motivated by the continuous decrease in malaria burden, the Thai government has declared a national malaria elimination plan intending to achieve this by the year 2024 [4].

To accomplish this goal within the time frame, a key strategy is to increase the bed net coverage and utilization, especially insecticide-treated nets (ITN) or long-lasting insecticidal nets (LLIN). The goal is to achieve $90 \%$ LLIN coverage among populations in high transmission areas (designated as A1 or A2 areas). A1 is village with reported indigenous malaria cases in current financial year and A2 is village without indigenous malaria cases for past 1-3 years [5]. Previous studies among the general population in Thailand and among the populations on the Thai-Myanmar border (Prachuap Khiri Khan Province) identified poor coverage and poor utilization of ITN/LLINs [6, 7]. However, actual coverage and utilization of bed nets as well as factors attributing to bed net use have not been investigated in this study area.

The objective of this study was to assess the ownership, accessibility, and utilization of both treated (ITNs and LLINs) and untreated bed nets in a remaining malaria transmission focus in western Thailand. A cross-sectional study was conducted and questionnaires were used to determine predictors of bed net use. The results are useful for formulating appropriate policies for the control programs and for the promotion of LLINs and long-lasting insecticide-treated hammock net (LLIHN) within the context of populations living along the malarious borders.

\section{Methods}

\section{Study design}

This is a mixed-methods cross-sectional study that included a questionnaire, an inspection form, and semi-structured interviews among selected participants. The household survey was conducted from August to October 2019.

\section{Study site}

A community-based cross-sectional survey was conducted in the areas under the International Center of Excellence for Malaria Research (ICEMR) project in Tha Song Yang District, Tak Province, northwestern Thailand (Fig. 1). Tha Song Yang is situated in the northwestern region of Tak, on the Moei River bank near the Myanmar border. The climate is tropical with an annual average temperature of $26.4^{\circ} \mathrm{C}$. The rainy season is between May and October, with an average yearly rainfall of $1540 \mathrm{~mm}$. The inhabitants of this 
area are approximately $30 \%$ Thai and $70 \%$ ethnic minorities. Normally, there are two peaks of malaria transmission, one at the beginning of the rainy season (May-August) and the other at the end of the rainy season (October) [8]. Plasmodium vivax and $P$. falciparum, respectively, are the predominant species in this region, although all human malaria parasites, as well as the simian malaria species $P$. knowlesi, have been identified in this area [9]. This study was conducted in 5 hamlets including Nong Bua, and Tala Oka (Mae Usu sub-district), Suan Oi, Pha Man, and Ko Ma Nae (Tha Song Yang sub-district).

\section{Study population, sampling and sample size}

For the quantitative component of the study, households residing in the study site were randomly selected from the study villages. Out of a total of 918 households in the 5 hamlets, 335 were selected by probability proportional to hamlet size, using an online statistical calculator [10]. Most of the household members are the Karen ethnic group [11]. From each household, the questionnaire and inspection form were administered to heads of household or representatives who look after the household. For the qualitative component, 24 potential respondents including 2 community leader from each sub-district, and 4 heads of household from each hamlet were selected to participate in the semi-structured interview. Purposive sampling was used to recruit participants for this part of the research. The target participation and the potential participants were selected based on geography, age, gender, and reported bed net use (including both those who do and don't report using bed nets). Important community leaders as "gatekeepers" were consulted and engaged in order to identify and invite the participants to join this study.

\section{Household surveys}

A community-based survey was conducted to assess the ownership, accessibility, and utilization of both treated (ITNs and LLINs) and untreated bed nets using a questionnaire, inspection form, and semistructured interviews. Respondents from 331 households in completed the interviews. In the selected households, face-to-face interviews were conducted with heads of household by a trained study team who speak and understand both Thai and Karen languages.

\section{Data analysis and statistics}

Data were extracted from the survey database and imported into the SPSS program version 22.0 [12] for analysis. Proportions (with 95\% confidence intervals) were used to summarize categorical variables related to ownership, accessibility, and utilization of bed nets. Log-binomial regression was used to explore the sociodemographic factors associated with bed net utilization ( $1=$ yes, used bed net; $0=$ no, did not use). Since populations in the same household are likely to have the same pattern of either using or not using bed nets, the log-binomial regression model included a random intercept for household to account for confounding and difference in response variation within and between households. An adjusted prevalence ratio (PR) (with 95\% confidence intervals [Cls]) was used to measure the strength of this association. We used log-binomial regression (calculating adjusted PRs) rather than logistic 
regression (and calculating model-adjused odds ratios) because the proportion of bed net use was high and may have resulted in misleading model adjusted odds ratios.

The qualitative analysis employed a thematic analysis approach [13]. The tentative code categories were given in the conceptual framework. Data were analyzed using thematic analysis of content to allow bringing together of similar views from different respondents together.

\section{Results}

\section{Household-level characteristics}

For the 331 households participating in the study, the majority had bamboo/wood walls (94.56\%), bamboo/wood floors (82.18\%), and terracotta/galvanized iron roofs (71.60\%) (Fig. 2). Among them, 315 (95.17\%) households were from the Karen ethnic minority, and $4.83 \%$ were Thai. Questionnaires were administered to $122(36.86 \%)$ male and 209 (63.14\%) female household heads. The mean age of the 331 respondents was 43.42 ( \pm 14.03$)$ years; $229(69.18 \%)$ heads of households were 35 years or older. A large proportion $(77.64 \%)$ of the respondents were illiterate. Over half $(54.98 \%)$ of the households had a family income of $\leq 2,000$ Baht/month (Table 1). For the 1,423 household members who reported bed net use, $787(55.31 \%)$ were female (Table 2). The mean age of the household members was $25.68( \pm 20.05)$ years; $620(43.57 \%)$ were 18-59 years old. For the participating household members, 186 (13.07\%) of whom stayed in the forests or the field. 
Table 1

Household and heads of household characteristics of border population in Tha Song Yang district, Tak province, Thailand

\begin{tabular}{|c|c|}
\hline Characteristics & Total $n=331 \mathrm{n}(\%)$ \\
\hline \multicolumn{2}{|l|}{ Wall } \\
\hline Bamboo/ wood & $313(94.56)$ \\
\hline Concrete & $18(5.44)$ \\
\hline \multicolumn{2}{|l|}{ Floor } \\
\hline Bamboo/ wood & $272(82.18)$ \\
\hline Concrete & $59(17.82)$ \\
\hline \multicolumn{2}{|l|}{ Roof } \\
\hline Thatch & $94(28.40)$ \\
\hline Terracotta/ galvanized iron & $237(71.60)$ \\
\hline \multicolumn{2}{|l|}{ Sex } \\
\hline Male & $122(36.86)$ \\
\hline Female & $209(63.14)$ \\
\hline \multicolumn{2}{|l|}{ Age (years) } \\
\hline$<35$ & $102(30.82)$ \\
\hline$\geq 35$ & $229(69.18)$ \\
\hline Mean $( \pm S D)$ & $43.42( \pm 14.03)$ \\
\hline \multicolumn{2}{|l|}{ Ethnics } \\
\hline Thai & $16(4.83)$ \\
\hline Karen & $315(95.17)$ \\
\hline \multicolumn{2}{|l|}{ Education level } \\
\hline Illiterate & $257(77.64)$ \\
\hline Literate & $74(22.36)$ \\
\hline \multicolumn{2}{|l|}{ Family income/month (Bath) } \\
\hline$\leq 2,000$ & $182(54.98)$ \\
\hline$>2,000$ & $149(45.02)$ \\
\hline Mean $( \pm S D)$ & $2630.51( \pm 1321.23)$ \\
\hline
\end{tabular}


Table 2

Household member characteristics of border population in Tha Song Yang district, Tak province, Thailand

\begin{tabular}{|ll|}
\hline Characteristics & Total $\mathbf{n}=1,423 \mathbf{n}(\%)$ \\
\hline Sex & $636(44.69)$ \\
\hline Male & $787(55.31)$ \\
\hline Age (years) & \\
\hline$\leq 10$ & $428(30.08)$ \\
\hline $11-17$ & $263(18.48)$ \\
\hline $18-59$ & $620(43.57)$ \\
\hline$\geq 60$ & $112(7.87)$ \\
\hline Mean $( \pm$ SD) & $25.68( \pm 20.05)$ \\
\hline Household member stay overnight outside in the forest or the field & \\
\hline Yes & $186(13.07)$ \\
\hline No & $1,237(86.93)$ \\
\hline
\end{tabular}

\section{Bed net ownership, access, and utilization}

Table 3 shows the household-level bed net ownership, access, and utilization. Almost all (98.49\%) households had at least one bed net per household, and $74.32 \%$ had at least one ITNs/LLINs in the household. However, only $30.82 \%$ of all households had sufficient numbers of ITN/LLINs, as per standard policy i.e., one ITN/LLIN per two persons. Moreover, only $4.30 \%$ of forest goers had owned LLIHNs. Besides, $86.10 \%$ of the households had sufficient numbers of bed net to cover all sleeping spaces. Although $94.17 \%$ of the household members slept in the sleeping spaces with any type of bed nets, $63.46 \%$ slept in sleeping spaces with ITNs or LLINs. 
Table 3

Household ownership access and utilization of bed nets among border population in Tha Song Yang district, Tak province, Thailand

\begin{tabular}{|l|l|}
\hline Characteristics & $\mathbf{n}(\%)$ \\
\hline Household ownership of bed nets & $326(98.49)$ \\
\hline At least one net per household (any type) & $246(74.32)$ \\
\hline At least one ITN/LLIN per household & \\
\hline Access of bed nets & $168(50.76)$ \\
\hline One net per two people (any type) & $102(30.82)$ \\
\hline One ITN/LLIN per two people & $8(4.30)$ \\
\hline One LLIHN per one people & $1,340(94.17)$ \\
\hline$\%$ of population with access to any bed net & $903(63.46)$ \\
\hline$\%$ of population with access to ITN/LLIN & $285(86.10)$ \\
\hline$\%$ of household have sufficient numbers of bed net to cover & \\
\hline all sleeping spaces & $1,311(92.13)$ \\
\hline Utilization of bed nets & $881(61.91)$ \\
\hline$\%$ of population slept under any bed net last night & $122(65.59)$ \\
\hline$\%$ of population slept under an ITN/LLIN last night & $1,151(80.89)$ \\
\hline$\%$ of forest goers slept under an ITN/LLIN last night & $756(53.13)$ \\
\hline$\%$ of population slept under any bed net every day & $16(8.60)$ \\
\hline$\%$ of population slept under an ITN/LLIN every day & $408(95.33)$ \\
\hline$\%$ of forest goers slept under an ITN/LLIN every day & $255(59.58)$ \\
\hline$\%$ of children under 10 years slept under any bed net last night & $10(100.00)$ \\
\hline$\%$ of children under 10 years slept under an ITN/LLIN last night & $9(90.00)$ \\
\hline$\%$ of pregnant women slept under any bed net last night & \\
\hline$\%$ of pregnant women slept under an ITN/LLIN last night & \\
\hline
\end{tabular}

Most of the household members (92.13\%) reported using bed nets in the previous night, and 881 (61.91\%) used ITNs or LLINs (Table 3). Since some respondents did not use bed net every day, especially in the summer season or when staying overnight in the forests or agricultural fields, daily use of bed net was also recorded. While 1,151 (80.89\%) household members reported bed net usage every day, 756 
(53.13\%) used ITNs or LLINs. Only $8.60 \%$ of forest goers use ITN/LLIN every day. For children under 10 years $(n=428), 95.33 \%$ slept under a bed net in the previous night, and 59.58\% slept under an ITN/LLIN. Among the pregnant women $(n=10)$, this was $100.00 \%$ and $90.00 \%$, respectively. Out of all 601 inspected bet nets, 109 (18.14\%) had holes (defined as a tear or opening that a finger could fit through.).

The most common pattern of bed net deployment by the household heads included inspecting for holes (86.32\%) and checking for mosquitos trapped inside (83.06\%). However, only $36.81 \%$ tucked in the nets fully before sleeping, and $28.99 \%$ slept away from the edges of the bed. Only $103(33.55 \%)$ of the household heads deployed the bed nets properly (Table 4).

Table 4

Pattern of deployment of bed net by head of household owning and using bed net $(n=307)$

\begin{tabular}{|lll|}
\hline & n & $\%$ \\
\hline Pattern of deployment of bed nets ${ }^{\text {a }}$ & & \\
\hline Tucking in fully during sleeping & 113 & 36.81 \\
\hline Inspecting for holes & 265 & 86.32 \\
\hline Checking for mosquitos trapped inside & 255 & 83.06 \\
\hline Sleeping away from the edge of the bed & 89 & 28.99 \\
\hline Head of household deployed bed net properly & & \\
\hline Yes & 103 & 33.55 \\
\hline No & 204 & 66.45 \\
\hline Total & 307 & 100.00 \\
\hline aMultiple responds allowed & & \\
\hline
\end{tabular}

\section{Sociodemographic factors associated with bed nets usage}

Univariate log-binomial regressions were used to compare the daily bed nets use across the different variables. Based on the univariate models, stronger associations were found between bed net use and variables such as "stay overnight in the forest or the field" [no vs yes: $\mathrm{PR}=6.26,95 \% \mathrm{Cl}=4.14-9.46 ; \mathrm{p}<$ 0.001 ], "like to use free bed net" [yes or indifferent vs not like: $P R=1.81,95 \% \mathrm{Cl}=1.19-2.75 ; \mathrm{p}=0.006$ ], sufficient numbers of bed net to cover all sleeping spaces [yes vs no: $P R=1.60,95 \% \mathrm{Cl}=1.24-2.06 ; \mathrm{p}<$ 0.001], sleeping pattern based on gender [female sleep with female vs male sleep alone: $P R=1.56,95 \%$ $\mathrm{Cl}=1.07-2.27 ; \mathrm{p}=0.021]$, and age [18-59 vs $\leq 10: \mathrm{PR}=0.74,95 \% \mathrm{Cl}=0.61-0.89 ; \mathrm{p}=0.001]$. Additionally, a weak association was found between daily bed net use and marital status [others vs married: $P R=1.26$, 
$95 \% \mathrm{Cl}=1.07-1.48 ; \mathrm{p}=0.005$ ], sex [female vs male: $\mathrm{PR}=1.23,95 \% \mathrm{Cl}=1.05-1.44 ; \mathrm{p}=0.012$ ], and sleeping pattern based on age ( $\leq 10$ year) [sleep with child vs not sleep with child: $P R=1.20,95 \% \mathrm{Cl}=$ $1.02-1.41 ; p=0.03]$.

In the final multivariable log-binomial regression, all three explanatory variables including "not stay overnight in the forest or the field", "like to use free bed nets", and "sufficient numbers of bed net to cover all sleeping spaces" showed a significant association with daily bed net use (Table 5). 
Table 5

Variables related to use of bed nets every day among respondents living in households owning at least one net $(n=1,401)$

\begin{tabular}{|c|c|c|c|c|c|}
\hline & $\mathrm{n}(\%)$ & $\begin{array}{l}\text { Univariat } \\
\text { analysis }\end{array}$ & & $\begin{array}{l}\text { Multivar } \\
\text { analysis }\end{array}$ & \\
\hline & & $\begin{array}{l}\text { PR } \\
(95 \% \mathrm{Cl})\end{array}$ & p-value & $\begin{array}{l}\text { PR } \\
\text { (95\%Cl) }\end{array}$ & p-value \\
\hline Age of household & & & & & \\
\hline$\leq 10(n=418)$ & 405 & 1 & - & - & - \\
\hline & $(96.89)$ & & & & \\
\hline $11-17(n=259)$ & 220 & 0.87 & 0.237 & 0.91 & 0.490 \\
\hline & $(84.94)$ & $\begin{array}{l}(0.70- \\
1.01)\end{array}$ & & $\begin{array}{l}(0.68- \\
1.20)\end{array}$ & \\
\hline $18-59(n=613)$ & 442 & 0.74 & $0.001 *$ & 0.84 & 0.367 \\
\hline & & $\begin{array}{l}(0.61- \\
0.89)\end{array}$ & & $\begin{array}{l}(0.58- \\
1.22)\end{array}$ & \\
\hline$\geq 60(n=111)$ & 84 & 0.76 & 0.093 & 0.94 & 0.773 \\
\hline & & $\begin{array}{l}(0.56- \\
1.05)\end{array}$ & & $\begin{array}{l}(0.60- \\
1.46)\end{array}$ & \\
\hline Sex of household & & & & & \\
\hline Male $(n=625)$ & $\begin{array}{l}456 \\
(72.96)\end{array}$ & 1 & - & - & - \\
\hline Female $(n=776)$ & $\begin{array}{l}695 \\
(8956)\end{array}$ & 1.23 & $0.012^{\star}$ & 1.07 & 0.501 \\
\hline & & $\begin{array}{l}(1.05- \\
1.43)\end{array}$ & & $\begin{array}{l}(0.88- \\
1.31)\end{array}$ & \\
\hline Marital status of $h$ & & & & & \\
\hline Married $(n=610)$ & $\begin{array}{l}439 \\
(71.97)\end{array}$ & 1 & - & - & - \\
\hline Others $(\mathrm{n}=791)$ & 712 & 1.26 & $0.005^{\star}$ & 0.91 & 0.570 \\
\hline & & $\begin{array}{l}(1.07- \\
1.48)\end{array}$ & & $\begin{array}{l}(0.64- \\
1.28)\end{array}$ & \\
\hline $\begin{array}{l}\text { Household membe } \\
\text { the forest or the fie }\end{array}$ & & & & & \\
\hline Yes $(n=184)$ & $\begin{array}{l}27 \\
(14.67)\end{array}$ & 1 & - & - & - \\
\hline
\end{tabular}

${ }^{*} \mathrm{p}$-value $<0.05,{ }^{* \star} \mathrm{p}$-value $<0.001$ 


\begin{tabular}{|c|c|c|c|c|c|}
\hline & \multirow[t]{2}{*}{ n (\%) } & \multicolumn{2}{|c|}{$\begin{array}{l}\text { Univariate } \\
\text { analysis }\end{array}$} & \multicolumn{2}{|c|}{$\begin{array}{l}\text { Multivariable } \\
\text { analysis }\end{array}$} \\
\hline & & $\begin{array}{l}\text { PR } \\
(95 \% \mathrm{Cl})\end{array}$ & p-value & $\begin{array}{l}\text { PR } \\
(95 \% \mathrm{Cl})\end{array}$ & p-value \\
\hline \multirow[t]{2}{*}{ No $(n=1,217)$} & \multirow{2}{*}{$\begin{array}{l}1,124 \\
(92.36)\end{array}$} & 6.26 & \multirow{2}{*}{$<.001 * \star$} & 5.77 & \multirow{2}{*}{$<.001^{\star * *}$} \\
\hline & & $\begin{array}{l}(4.14- \\
9.46)\end{array}$ & & $\begin{array}{l}(3.76- \\
8.85)\end{array}$ & \\
\hline \multicolumn{6}{|l|}{ Head of household like to use free bed net } \\
\hline Not like $(n=75)$ & $\begin{array}{l}34 \\
(45.33)\end{array}$ & 1 & - & - & - \\
\hline \multirow[t]{2}{*}{ Yes or indifferent $(n=1,326)$} & \multirow{2}{*}{$\begin{array}{l}1,117 \\
(84.24)\end{array}$} & 1.81 & \multirow[t]{2}{*}{$0.006^{*}$} & 1.65 & \multirow[t]{2}{*}{$0.025^{\star}$} \\
\hline & & $\begin{array}{l}(1.19- \\
2.75)\end{array}$ & & $\begin{array}{l}(1.07- \\
2.55)\end{array}$ & \\
\hline \multicolumn{6}{|l|}{$\begin{array}{l}\text { Sufficient numbers of bed net to cover all } \\
\text { sleeping spaces in household }\end{array}$} \\
\hline No $(n=190)$ & $\begin{array}{l}104 \\
(54.74)\end{array}$ & 1 & - & - & - \\
\hline \multirow[t]{2}{*}{ Yes $(n=1,211)$} & \multirow{2}{*}{$\begin{array}{l}1047 \\
(86.46)\end{array}$} & 1.81 & \multirow{2}{*}{$<.001^{\star *}$} & 1.65 & \multirow[t]{2}{*}{$0.032^{\star *}$} \\
\hline & & $\begin{array}{l}(1.19- \\
2.75)^{\star}\end{array}$ & & $\begin{array}{l}(1.06- \\
2.55)^{\star}\end{array}$ & \\
\hline \multicolumn{6}{|l|}{ Sleeping pattern based on gender } \\
\hline Male sleep alone $(n=98)$ & $\begin{array}{l}61 \\
(62.24)\end{array}$ & 1 & - & - & - \\
\hline \multirow[t]{2}{*}{ Female sleep alone $(n=75)$} & \multirow[t]{2}{*}{$63(84)$} & 1.35 & \multirow[t]{2}{*}{0.201} & 1.11 & \multirow[t]{2}{*}{0.683} \\
\hline & & $\begin{array}{l}(0.85- \\
2.15)\end{array}$ & & $\begin{array}{l}(0.67- \\
1.86)\end{array}$ & \\
\hline \multirow[t]{2}{*}{ Male sleep with female (spouse) $(n=162)$} & \multirow{2}{*}{$\begin{array}{l}104 \\
(64.20)\end{array}$} & 1.04 & \multirow[t]{2}{*}{0.845} & 1.09 & \multirow[t]{2}{*}{0.719} \\
\hline & & $\begin{array}{l}(0.70- \\
1.56)\end{array}$ & & $\begin{array}{l}(0.69- \\
1.73)\end{array}$ & \\
\hline \multirow{2}{*}{$\begin{array}{l}\text { Male sleep with male ( } \geq 2 \text { person/sleeping } \\
\text { space) }(n=57)\end{array}$} & \multirow{2}{*}{$\begin{array}{l}43 \\
(75.44)\end{array}$} & 1.24 & \multirow[t]{2}{*}{0.402} & 1.14 & \multirow[t]{2}{*}{0.646} \\
\hline & & $\begin{array}{l}(0.75- \\
2.07)\end{array}$ & & $\begin{array}{l}(0.66- \\
1.95)\end{array}$ & \\
\hline \multirow{2}{*}{$\begin{array}{l}\text { Male sleep with female (not spouse) }(\geq 2 \\
\text { person/sleeping space) }(n=808)\end{array}$} & \multirow{2}{*}{$\begin{array}{l}687 \\
(85.02)\end{array}$} & 1.397 & \multirow[t]{2}{*}{0.052} & 1.26 & \multirow[t]{2}{*}{0.287} \\
\hline & & $\begin{array}{l}(0.99- \\
1.96)\end{array}$ & & $\begin{array}{l}(0.83- \\
1.91)\end{array}$ & \\
\hline
\end{tabular}




\begin{tabular}{|c|c|c|c|c|c|}
\hline & \multirow[t]{2}{*}{ n (\%) } & \multicolumn{2}{|c|}{$\begin{array}{l}\text { Univariate } \\
\text { analysis }\end{array}$} & \multicolumn{2}{|c|}{$\begin{array}{l}\text { Multivariable } \\
\text { analysis }\end{array}$} \\
\hline & & $\begin{array}{l}\text { PR } \\
(95 \% \mathrm{Cl})\end{array}$ & p-value & $\begin{array}{l}\text { PR } \\
(95 \% \mathrm{Cl})\end{array}$ & p-value \\
\hline \multirow{2}{*}{$\begin{array}{l}\text { Female sleep with female }(\geq 2 \\
\text { person/sleeping space) }(n=201)\end{array}$} & \multirow{2}{*}{$\begin{array}{l}193 \\
(96.02)\end{array}$} & 1.56 & \multirow[t]{2}{*}{$0.021^{\star}$} & 1.25 & \multirow[t]{2}{*}{0.341} \\
\hline & & $\begin{array}{l}(1.07- \\
2.27)\end{array}$ & & $\begin{array}{l}(0.79- \\
1.99)\end{array}$ & \\
\hline \multicolumn{6}{|l|}{ Sleeping pattern based on age ( $\leq 10$ year) } \\
\hline Not sleep with child $\leq 10$ year $(n=533)$ & $\begin{array}{l}394 \\
(73.92)\end{array}$ & 1 & - & - & - \\
\hline \multirow[t]{2}{*}{ Sleep with child $\leq 10$ year $(n=868)$} & \multirow{2}{*}{$\begin{array}{l}757 \\
(87.21)\end{array}$} & 1.20 & \multirow[t]{2}{*}{$0.030^{*}$} & 0.96 & \multirow[t]{2}{*}{0.753} \\
\hline & & $\begin{array}{l}(1.02- \\
1.41)\end{array}$ & & $\begin{array}{l}(0.72- \\
1.27)\end{array}$ & \\
\hline
\end{tabular}

Log-binomial regression was also used to compare the bed net use among respondents who stayed overnight outside the house in the previous year, but only family income showed a significant association with daily bed net use in the final multivariable log-binomial regression (data not tabulated).

\section{Reasons for use or non-use of bed nets in the household and the forest}

A total of 22 respondents were interviewed by semi-structured interview. The results organized into key themes that emerged from the discussions.

\section{Reasons for use of bed nets in the household}

Most participants reported using bed nets to prevent mosquito bites and malaria infection. Most of them remembered using bed nets from a very young age, whereas some of them started to use bed nets when they had children or grandchildren. Bed net use had become a habit for them and they could not sleep without it.

"I use bed nets to protect myself against mosquito bites. I have used bed nets since I was born and becoming my habit I cannot sleep without the net" (Female villager, Mae Usu subdistrict)

Additionally, free bed net distribution campaigns were mentioned as a factor that supported them to start and continue using bed nets in the last 20-30 years.

"After I immigrated to Thailand 20 years ago, I started to use bed nets and they were free bed nets distributed from health providers. The net is good and prevents mosquitoes from biting and not getting 
sick with malaria." (Female villager, Tha Song Yang subdistrict)

\section{Bed net use in farms and forests}

Forest goers in this area had not used any kinds of bed net while they were in the forest. Most forest goers used bed net only in the subsistence farm huts, their temporary residence nearby the forest. Both forest goers and farmers, if they had sufficient bed nets for use in their households, they would take the old bed nets for use in the subsistence farm huts. If they had limited bed nets, they would carry bed net to the subsistence farm hut and carry it back home, especially when children accompanied them to the farms. However, many of them used bed nets in a subsistence farm hut only in the rainy season when mosquitoes are abundant.

"When children accompanied us to the farm plots or forest, we always carried bed net to use in the farm hut. Our children need to get protection from mosquito bites." (Female villager, Mae Usu subdistrict)

"I sleep under bed net every day. And even when I was going to overnight in the farm, I took an old bed net to use in the farm hut." (Male villager, Tha Song Yang subdistrict)

\section{Reasons for non-use of bed nets in the household}

The major reason that participants reported for not using bed nets every day at home was discomfort from the heat and perception of unnecessity due to low mosquito density, especially in the hot season.

"I am still afraid of malaria, but I could not sleep it was too hot to sleep under the nets in the summer" (Female villager, Tha Song Yang subdistrict)

The second most commonly reported reason was feeling complacent and not wanting to expend the effort needed to set up and use nets in the evenings, especially for adolescents who did not share sleeping space with their parents.

"My son and daughter rarely use bed net because they are so lazy to hang the bed net up. I sometimes have to hang it for them and tell them to sleep under bed net." (Female villager, Mae Usu subdistrict)

Other reasons mentioned for not using a net at home included inadequate number of bed nets, being not habitual of sleeping under the bed nets, use of alternative mosquito control methods, too small size of the free bed net, rough texture of material of free bet nets, strong smell of insecticide, and inadequate space to hang a bed net.

\section{Reasons for non-use of bed nets in the forest or farms}

The major reason for the non-use of bed nets in the forest was inconvenient to carry the bed nets and prioritized other essential items. Even in the subsistence farm hut, some felt lazy to unpack and hang the bed nets up before sleeping in. Although someone received an LLIHN to use in the forest, they tried to use the hammocks in the forest, they found problems from using the net. 
"Going to forest, we travel light with a few necessary items so we can carry lots forest products when we return. It is inconvenient to bring the net to the forest, it takes space to carry. It also can get entangled with other items, obstructed hunting and gatering process, in particular to hunting we need to be quick to shoot the targets. Sometime at night, animals such as elephants approach our sleeping area, we have to run away, we have no time to pack the net." (Male villager, Tha Song Yang subdistrict)

The second reason was the inadequate number of the nets for use when visiting the forest. Other reasons for not using a net in the forest included being not habitual of sleeping under the bed nets, discomfort from heat, and use of alternative mosquito control methods.

\section{Discussion}

This is the first cross-sectional study assessing the ownership and utilization of bed nets among the border population in Tha Song Yang District of western Thailand. The results of this study revealed that the ownership of bed nets is very high (98.49\%), while 246 (74.32\%) had at least one treated nets. Bed nets are highly available in these localities, a result of efforts by the Department of disease control and other partners (such as non-governmental organizations) that have distributed free LLINs from the Global Fund [6]. The results are consistent with the finding of the Mobile and Migrant Population Survey in Thailand [6], which found that households with any nets were $94 \%$ in 2017, whereas households with at least one ITN were 39\%. Also, Kitidamrongsuk et al [7], reported that almost all households (92.9\%) in Prachuab Khiri Khan, Thailand had at least one mosquito net, whereas $41.1 \%$ of households owned at least one ITN/LLIN. Similar studies in Myanmar showed high coverage of bed nets among households of Kachin Special Region II population [14], and 50-65\% coverage of ITN/LLIN among households of migrant population $[15,16]$. The WHO advocates for universal coverage of ITN/LLINs with one net for every 2 persons [17]. In spite of the high-level ownership of and access to bed nets in this study because of the LLIN distribution campaign, only $30.82 \%$ of all households had sufficient numbers of ITN/LLIN for one ITN/ LLINs per two persons. This insufficiency might be due to the operational challenges of the LLIN distribution system, as some studies identified operational barriers to continuous LLIN distribution [18, 19]. This study suggests that the insufficiency of ITN/LLIN is also a concern and can be used as an indicator for the LLIN distribution program. Plucinski et al. reported that the access indicators of LLINs were high in Mozambique when the bed net distribution campaigns used a novel distribution model and used LLINs designated for each sleeping space [20]. Thus, the LLIN distribution systems in Thailand should be evaluated in the future.

Globally, malaria-related morbidity and mortality are highest in children and pregnant women [21]. Most children in this study used bed net in the previous night (95.3\%). Overall bed net usage among adults was lower than among children. This was mainly due to the high focus on vulnerable age groups. However, only $59.58 \%$ of the children used ITNs/LLINs. This result is consistent with the previous findings in Myanmar and some countries in Africa $[6,15,16,22-26]$, which showed that the utilization of ITN remained moderate or low among children. However, it differs from the previous reports of high use rate of ITNs/LLINs among children $[14,27,28]$. Pregnant women, particularly primigravidas with malaria, have 
a high risk of severe malaria and low birth weights $[29,30]$. This study showed a high rate of ITN/LLIN use among pregnant women $(90.00 \%)$. However, in many parts of the world, ITN usage remained moderate or low among pregnant women $[6,16,22,23,28,31]$.

One of the major factors in not using bed nets in our data was the use of different sleeping sites. Work and evening activities in the forest impact where community members choose to sleep on any particular evening. Together with the previous findings in Prachuab Khiri Khan province [7] and Southern Thailand [32], sleeping elsewhere especially at temporary shelters in the plantation is another non-use reason.

Another major factor in bed net use was net manufacturing materials and size are other factors affecting net utilization among households with more family members [7]. Many other studies reported under usage of nets due to the lack of sufficient nets to cover all household members [7, 15, 26, 27, 33-35]. Household socio-economic status has likewise been shown to influence bed net access and is the strongest determinant of net use [28, 36-43]. Our study showed that wealthier families were significantly more likely to use bed nets every day than the poorer families, likely because of the ability to purchase bed nets for use outside the home.

Consistent with previous studies [44-47],the perceived role of bed nets as a means to protect against mosquitoes and malaria was a significant predictor of seasonal bed net use. In our research, participants reported higher bed net usage during the rainy season. Aside from malaria prevention, the main reason for sleeping under nets was because they provided comfortable sleep and protected against biting insects (including mosquitoes).

Other reasons for the not using bed nets were in line with other research $[7,16,48-57]$ suggesting that discomfort from heat and low mosquito density are common reasons for non-adherence. Complacence, the inadequate number of of bed net, rough materials of bed net, not having a routine of bed net use, use of alternative mosquito control methods, and too small size of free bed nets were the other most commonly cited reasons for not using a net at home. Some studies have shown that bed nets decrease airflow, making it feel hot and stifling under a net $[48,58,59]$. In seasons of low and/or variable mosquito nuisance, education will need to emphasize that the risk of malaria is not necessarily diminished when mosquito numbers are perceived to be low.

The reasons for not using bed net in the forest were also consistent with some past studies in the greater Mekong subregion [60-62]. Some forest-goers described the inconvenience of carrying bed nets, while others found inadequacy of nets for use when visiting the forest. This study suggests that the campaign of LLIHN distribution appeared not effective for malaria prevention among forest goers. The advantages of these nets might be not adequately promoted. Other strategies to address outdoor transmission may need to be considered among this population [63-65].

Previously reported limited effectiveness of bed nets in Southeast Asia may have resulted from the vector feeding behavior and human activities that in some circumstances increase human-vector contacts [66, 67]. Some main malaria vectors such as Anopheles minimus, Anopheles maculatus and Anopheles dirus 
feed outdoors at dusk between 6 and 7 p.m. when people are not in bed $[66,68]$. Also, forest goers exhibit behaviors (hunting or fishing) that reduce the protection of ITNs at peak biting times [69, 70]. Despite high coverage and utilization of bed nets in this study, the bed net use behavior of people may also affect the effectiveness of bed nets. Some did not use bed net every day, especially in summers and when they stayed overnight in the forest or the field. Some did not deploy bed nets properly, used damaged/torn nets, and used untreated nets. Nevertheless, given the broader impacts of ITNs for preventing all vectorborne diseases such as malaria [71, 72], Japanese encephalitis [73], and leishmaniasis [74], proper net distribution and encouraged use still need to be strengthened, especially for the high-risk populations such as the forest goers.

Outdoor transmission remains the main challenge for malaria elimination program in Thailand. This transmission is influenced by human behavior, including efforts at preventing exposure to mosquito vectors. At least one study (from Tanzania) has shown that relatively modest coverage (53.13\% ITNs use) in the community can lead to community-wide protection against malaria [75]. However high risk groups such as Karen ethnic groups living along the border and forest goers may not receive the same benefits of the protection. In our study, low use of bed nets when overnighting in the forest was related to perceptions of inconvenience of carrying and using bed nets, insufficient numbers of bed nets to use outside the home, and low perception of bed net necessity. Therefore, interventions among these high risk groups should pay attention to factors beyond bed net coverage, and use and look for long-lasting new tools or approaches for preventing exposure to mosquito vectors to decrease outdoor malaria transmission.

Study limitations included $63.14 \%$ of heads of households were female. However, female heads of households should be in better position to respond about ownership and use of bed nets of every household member. This study was conducted in Thasongyang district; therefore, the results could represent population living along Thai-Myanmar border and Karen ethnic groups, but not represent Karen groups from other areas in which lifestyle, culture, or health operation were different. Due to the limitation of budget and time, this study was conducted in one season (rainy season with more abundant of mosquitoes) which may result in high rate of bed net use in the previous night. Nonetheless, adding question of everyday use of bed net, should reduce the bias from season differences.

\section{Conclusions}

This study showed that high overall coverage and usage of bed nets in the study area; however, only one third reached the standard level specified by the policy. Overnighting in the forest or the farm plots, the dissatisfaction with the quality of free bed nets, insufficient number of bed nets, discomfort from heat, perception of no benefits of bed nets due to low mosquito density, and inconvenience were factors influencing bed net use. Moreover, only one third of heads of households deployed bed net properly. Further health promotion programs should ensure to maintain high coverage and utility rate of bed nets and further studies should prioritize the investigation of new preventive tools for effective outdoor protection in particualt ot the forest goers. 


\section{Abbreviations}

ITN: insecticide-treated net; LLIN: long-lasting insecticidal net; LLIHN: long-lasting insecticide-treated hammock net; ICEMR: International Center of Excellence for Malaria Research; PR: prevalence ratio; Cl: confidence interval.

\section{Declarations}

\section{Acknowledgments}

We thank all participating community member, community leaders, and health staffs for their dedicated study participation.

\section{Authors' contributions}

$\mathrm{KP}, \mathrm{SP}$, and PS conceived and designed the study. KP and SP collected data and conducted interviews. KP and SL participated in sample processing and data analysis. KP, DMP, NM, SL, PS, JS, LC, and SP participated in data analysis, interpretation, and drafting the manuscript. All authors read and approved the fnal manuscript.

\section{Funding}

This study was funded by the National Institute of Allergy and Infectious Diseases, National Institutes of Health (U19 Al089672) and Faculty of Tropical Medicine, Mahidol University, Thailand.

\section{Availability of data}

The datasets used and/or analysed during the current study are not provided.

\section{Ethics approval and consent to participate}

The heads of households or adult representatives were approached for consenting before recruitment. A written informed consent was obtained as permission to conduct data collection. The study was approved by the Ethics Committee of the Faculty of Tropical Medicine, Mahidol University, Thailand (Ethical number MUTM 2019-056-01).

\section{Consent for publication}

All authors gave the consent for this publication.

\section{Competing interests}

The authors declare that they have no competing interests.

\section{Author details}


${ }^{1}$ Department of Tropical Hygiene, Faculty of Tropical Medicine, Mahidol University, Thailand

${ }^{2}$ Faculty of Public Health, Thammasat University, Thailand

${ }^{3}$ Department of Population Health \& Disease Prevention, Susan and Henry Samueli College of Health Sciences, Program in Public Health, University of California, Irvine, USA

${ }^{4}$ Department of Society and Health, Faculty of Social Sciences and Humanities, Mahidol University, Thailand

${ }^{5}$ Mahidol Vivax Research Unit, Faculty of Tropical Medicine, Mahidol University, Thailand

${ }^{6}$ Department of Internal Medicine, Morsani College of Medicine, University of South Florida, Tampa, FL, USA

${ }^{7}$ SEAMEO TROPMED Regional Centre for Tropical Medicine, Faculty of Tropical Medicine, Mahidol University, Thailand

${ }^{8}$ Department of Social and Environmental Medicine, Faculty of Tropical Medicine, Mahidol University, Thailand

\section{References}

1. WHO. World malaria report 2020. Geneva, World Health Organization, 2020.

2. Division of Vector-Borne Diseases, Department of Disease Control. Ministry of Public Health, Thailand. Database of Thailand malaria elimination program. http://malaria.ddc.moph.go.th/malariaR10/index_v2.php. Accessed 5 Mar 2021.

3. Division of Vector-Borne Diseases, Department of Disease Control. Ministry of Public Health, Thailand. Annual Report Malaria situation in Thailand 2018. Nonthaburi, Ministry of Public Health, 2018.

4. Division of Vector-Borne Disease, Department of Disease Control. Ministry of Public Health, Thailand. National Strategic Plan for Malaria Control and Elimination in Thailand 2017-2026. Nonthaburi, Ministry of Public Health, 2017.

5. Division of Vector-Borne Diseases, Department of Disease Control. Ministry of Public Health, Thailand. A malaria elimination operational manual for Communicable Disease Control Unit and Surveillance and Rapid Response Team. Nonthaburi, Ministry of Public Health, 2019.

6. President's malaria initiative Thailand. Lao PDR, and regional. Malaria Operational Plan FY 2018. 2018.

7. Kitidamrongsuk P, Jareinpituk S, Pattanasin S, Viwatwongkasem C, Soontornpipit P, Silabutra J, et al. Determinants of Impregnated Net Ownership and Utilization in Rural Community on the ThaiMyanmar Border in Prachuab Khiri Khan, Thailand. Procedia Comput Sci. 2016;86:224-7. 
8. Cui L, Mascorro CN, Fan Q, Rzomp KA, Khuntirat B, Zhou G, et al. Genetic diversity and multiple infections of Plasmodium vivax malaria in Western Thailand. Am J Trop Med Hyg. 2003;68:613-9.

9. Baum E, Sattabongkot J, Sirichaisinthop J, Kiattibutr K, Jain A, Taghavian O, et al. Common asymptomatic and submicroscopic malaria infections in Western Thailand revealed in longitudinal molecular and serological studies: a challenge to malaria elimination. Malar J. 2016;15:333.

10. Dhand NK, Khatkar MS. Statulator. An online statistical calculator. Sample Size Calculator for Comparing Two Independent Proportions. http://statulator.com/SampleSize/ss2P.html. Accessed 20 Apr 2019.

11. Lawpoolsri S, Sattabongkot J, Sirichaisinthop J, Cui L, Kiattibutr K, Rachaphaew N, et al. Epidemiological profiles of recurrent malaria episodes in an endemic area along the ThailandMyanmar border: a prospective cohort study. Malar j. 2019;18:124.

12. IBM Corp. Released. IBM SPSS Statistics for Windows, Version 22.0. Armonk. NY: IBM Corp; 2013.

13. Braun V, Clarke V. Using thematic analysis in psychology. Qual Res Psychol. 2006;3:77-101.

14. Liu H, Xu J, Guo X, Havumaki J, Lin Y, Yu G, et al. Coverage, use and maintenance of bed nets and related influence factors in Kachin Special Region II, northeastern Myanmar. Malar J. 2015;14:212.

15. Maung TM, Tripathy JP, Oo T, Oo SM, Soe TN, Thi A, et al. Household ownership and utilization of insecticide-treated nets under the Regional Artemisinin Resistance Initiative in Myanmar. Trop Med Health. 2018;46:27.

16. Linn SY, Maung TM, Tripathy JP, Shewade HD, Oo SM, Linn Z, et al. Barriers in distribution, ownership and utilization of insecticide-treated mosquito nets among migrant population in Myanmar, 2016: a mixed methods study. Malar j. 2019;18:172.

17. Evaluation MEASURE, MEASURE DHS, President's Malaria Initiative, Roll Back Malaria Partnership, UNICEF, World Health Organization. Household Survey Indicators for Malaria Control. 2013.

18. Theiss-Nyland K, Kone D, Karema C, Ejersa W, Webster J, Lines J. The relative roles of ANC and EPI in the continuous distribution of LLINs: a qualitative study in four countries. Health Policy Plan. 2017;32:467-75.

19. Webster J, Kayentao K, Diarra S, Diawara SI, Haiballa AA, Doumbo OK, Hill JA. qualitative health systems effectiveness analysis of the prevention of malaria in pregnancy with intermittent preventive treatment and insecticide treated nets in Mali. PloS One. 2013;8:e65437-7.

20. Plucinski MM, Chicuecue S, Macete E, Colborn J, Yoon SS, Kachur SP, et al. Evaluation of a universal coverage bed net distribution campaign in four districts in Sofala Province, Mozambique. Malar J. 2014;13:427.

21. Schantz-Dunn J, Nour NM. Malaria and pregnancy: a global health perspective. Rev Obstet Gynecol. 2009;2:186-92.

22. Inungu JN, Ankiba N, Minelli M, Mumford V, Bolekela D, Mukoso B, et al. Use of Insecticide-Treated Mosquito Net among Pregnant Women and Guardians of Children under Five in the Democratic Republic of the Congo. Malar Res Treat. 2017;2017:5923696-. 
23. Doda Z, Solomon T, Loha E, Gari T, Lindtjørn B. A qualitative study of use of long-lasting insecticidal nets (LLINs) for intended and unintended purposes in Adami Tullu, East Shewa Zone, Ethiopia. Malar j. 2018;1:69.

24. Diema KK, Japiong M, Dodam KK, Afaya A, Salia SM, Kombat JM. Utilization of Insecticide Treated Bed Nets (ITNs) among Caregivers of Children under Five Years in the Ho Municipality. Interdiscip Perspect Infect Dis. 2019;2019:3693450.

25. Admasie A, Zemba A, Paulos W. Insecticide-Treated Nets Utilization and Associated Factors among under-5 Years Old Children in Mirab-Abaya District, Gamo-Gofa Zone, Ethiopia. Front Public Health. 2018;6:7.

26. Min KT, Maung TM, Oo MM, Oo T, Lin Z, Thi A, et al. Utilization of insecticide-treated bed nets and care-seeking for fever and its associated socio-demographic and geographical factors among underfive children in different regions: evidence from the Myanmar Demographic and Health Survey, 2015-2016. Malar j. 2020;19:7.

27. Raghavendra K, Chourasia MK, Swain DK, Bhatt RM, Uragayala S, Dutta GDP, et al. Monitoring of long-lasting insecticidal nets (LLINs) coverage versus utilization: a community-based survey in malaria endemic villages of Central India. Malar j. 2017;16:467.

28. Xu JW, Liao YM, Liu H, Nie RH, Havumaki J. Use of bed nets and factors that influence bed net use among Jinuo Ethnic Minority in southern China. PLoS One. 2014;9:e103780.

29. Tsuang A, Lines J, Hanson K. Which family members use the best nets? An analysis of the condition of mosquito nets and their distribution within households in Tanzania. Malar J. 2010;9:211.

30. Desai MR, Kuile FO, Nosten F, McGready R, Asamoa K, Brabin BJ, et al. Epidemiology and burden of malaria in pregnancy. Lancet Infect Dis. 2007;7:93-104.

31. Fuge TG, Ayanto SY, Gurmamo FL. Assessment of knowledge, attitude and practice about malaria and ITNs utilization among pregnant women in Shashogo District, Southern Ethiopia. Malar J. 2015;14:235.

32. Pattanasin S, Satitvipawee P, Wongklang W, Viwatwongkasem C, Bhumiratana A, Soontornpipit P, et al. Risk factors for malaria infection among rubber tappers living in a malaria control program area in southern Thailand. Southeast Asian J Trop Med Public Health. 2012;43:1313-25.

33. Biadgilign S, Reda A, Kedir H. Determinants of ownership and utilization of insecticide-treated bed nets for malaria control in eastern Ethiopia. J Trop Med. 2012;2012:235015.

34. Moscibrodzki P, Dobelle M, Stone J, Kalumuna C, Chiu YM, Hennig N. Free versus purchased mosquito net ownership and use in Budondo sub-county, Uganda. Malar J. 2018;17:363.

35. Kateera F, Ingabire CM, Hakizimana E, Rulisa A, Karinda P, Grobusch MP, et al. Long-lasting insecticidal net source, ownership and use in the context of universal coverage: a household survey in eastern Rwanda. Malar J. 2015;14:390.

36. Sangare LR, Weiss NS, Brentlinger PE, Richardson BA, Staedke SG, Kiwuwa MS, et al. Determinants of use of insecticide treated nets for the prevention of malaria in pregnancy in Jinja, Uganda. PLoS One. 2012;7:e39712. 
37. Hetzel MW, Gideon G, Lote N, Makita L, Siba PM. Ownership and usage of mosquito nets after four years of large-scale free distribution in Papua New Guinea. Malar J. 2012;11:192.

38. Bowen HL. Impact of a mass media campaign on bed net use in Cameroon. Malar J. 2013;12:36.

39. Koenker H, Kilian A. Recalculating the net use gap: a multi-country comparison of ITN use versus ITN access. PLoS One. 2014;9:e97496.

40. Kanyangarara M, Hamapumbu H, Mamini E, Lupiya J, Stevenson JC, Mharakurwa S, et al. Malaria knowledge and bed net use in three transmission settings in southern Africa. Malar j. 2018;17:41.

41. Singh M, Brown G, Rogerson SJ. Ownership and use of insecticide-treated nets during pregnancy in sub-Saharan Africa: A review. Malar J. 2013;12:1-10.

42. Sena LD, Deressa WA, Ali AA. Predictors of long-lasting insecticide-treated bed net ownership and utilization: evidence from community-based cross-sectional comparative study, Southwest Ethiopia. Malar J. 2013;12:406.

43. Eteng M, Mitchell S, Garba L, Ana O, Liman M, Cockcroft A, et al. Socio-economic determinants of ownership and use of treated bed nets in Nigeria: Results from a cross-sectional study in Cross River and Bauchi States in 2011. Malar J. 2014;13:1-8.

44. Msellemu D, Shemdoe A, Makungu C, Mlacha Y, Kannady K, Dongus S, et al. The underlying reasons for very high levels of bed net use, and higher malaria infection prevalence among bed net users than non-users in the Tanzanian city of Dar es Salaam: a qualitative study. Malar j. 2017;16:423.

45. Koenker H, Munoz B, Boulay M, Mkocha H, Levens J, West SK, et al. Trends in weekly reported net use by children during and after rainy season in central Tanzania. Malar J. 2012;11:218.

46. Vanden EJ, Thwing J, Wolkon A, Kulkarni MA, Manya A, Erskine M, et al. Assessing bed net use and non-use after long-lasting insecticidal net distribution: a simple framework to guide programmatic strategies; insecticide-treated bed nets and curtains for preventing malaria. Malar J. 2010;9:133.

47. Atieli HE, Zhou G, Afrane Y, Lee M-C, Mwanzo I, Githeko AK, et al. Insecticide-treated net (ITN) ownership, usage, and malaria transmission in the highlands of western Kenya. Parasit Vectors. 2011;4:113.

48. Koenker HM, Loll D, Rweyemamu D, Ali AS. A good night's sleep and the habit of net use: perceptions of risk and reasons for bed net use in Bukoba and Zanzibar. Malar J. 2013;12:203.

49. Pulford J, Hetzel MW, Bryant M, Siba PM, Mueller I. Reported reasons for not using a mosquito net when one is available: a review of the published literature. Malar J. 2011;10:83.

50. Beer N, Ali AS, Eskilsson H, Jansson A, Abdul-Kadir FM, Rotllant-Estelrich G. et. al. A qualitative study on caretakers' perceived need of bed-nets after reduced malaria transmission in Zanzibar Tanzania. BMC Publ Health. 2012;12:606.

51. Adaji J, Gabriel OE. Access and Usag e of Long Lasting Insecticidal Nets (LLIN) in rural Communities of Benue State, Nigeria. Health Sci J. 2019;3:1-4.

52. Adebayo AM, Akinyemi 00, Cadmus EO. Ownership and of mosquito nets among caregivers of fi children and pregnant women in a rural community in Southwest Nigeria. J Prev Med Hyg. 
2014;55:58-64.

53. Iwashita H, Dida G, Futami K, Sonye G, Kaneko S, Horio M, et al. Sleeping arrangement and house structure affect bed net use in villages along Lake Victoria. Malar j. 2010;9:176.

54. Solomon T, Loha E, Deressa W, Gari T, Overgaard HJ, Lindtjørn B. Low use of long-lasting insecticidal nets for malaria prevention in south-central Ethiopia: A community-based cohort study. PLoS One. 2019;14:e0210578.

55. Wangdi K, Gatton ML, Kelly GC, Clements AC. Prevalence of asymptomatic malaria and bed net ownership and use in Bhutan, 2013: a country earmarked for malaria elimination. Malar j. 2014;13:352.

56. Nonaka D, Pongvongsa T, Nishimoto F, Nansounthavong P, Sato Y, Jiang H, et al. Households with Insufficient Bednets in a Village with Sufficient Bednets: Evaluation of Household Bednet Coverage Using Bednet Distribution Index in Xepon District, Lao PDR. Trop Med Health. 2015;43:95-100.

57. Monroe A, Asamoah O, Lam Y, Koenker H, Psychas P, Lynch M, et al. Outdoor-sleeping and other night-time activities in northern Ghana: implications for residual transmission and malaria prevention. Malar j. 2015;14:35.

58. Von SL, Ikonomidis K, Bruu R, Jawara M, Pinder M, Knols BGJ, et al. Airflow attenuation and bed net utilization: observations from Africa and Asia. Malar J. 2012;11:200.

59. Von SL, Ikonomedis K, Mshamu S, Nkya TE, Mukaka M, Pell C,et al. Affordable house designs to improve health in rural Africa: a field study from northeastern Tanzania. Lancet Planet Health. 2017;1:e188-99.

60. Grietens KP, Xuan XN, Van BW, Duc TN, Ribera JM, Nhat TB, et al. Low perception of malaria risk among the Ra-glai ethnic minority in southcentral Vietnam: implications for forest malaria control. Malar J. 2010;9:23.

61. Lyttleton C. Deviance and resistance: malaria elimination in the greater Mekong subregion. Soc Sci Med. 2016;150:144-52.

62. Wharton-Smith A, Shafique MA. Qualitative study to assess consumer preferences and barriers to use of long lasting insecticidal nets in Myanmar. London: Malaria Consortium; 2014.

63. Eisele TP, Bennett A, Simlumbe K, et al. The impact of targeted mass drug administration using dihydroartemisinin-piperaquine in Southern Province Zambia: initial findings. Philadelphia, PA: Am J Trop Med Hyg. 2015.

64. Committee WHOMPA, Secretariat. Malaria Policy Advisory Committee to the WHO: conclusions and recommendations of eighth biannual meeting (September 2015). Malar J. 2016;15:117.

65. Govella NJ, Ferguson $\mathrm{H}$. Why use of interventions targeting outdoor biting mosquitoes will be necessary to achieve malaria elimination. Front Physiol. 2012;3:199.

66. Smithuis FM, Kyaw MK, Phe UO, van der Broek I, Katterman N, Rogers C, et al. The effect of insecticide-treated bed nets on the incidence and prevalence of malaria in children in an area of unstable seasonal transmission in western Myanmar. Malar J. 2013;12:363. 
67. Edwards HM, Sriwichai P, Kirabittir K, Prachumsri J, Chavez IF, Hii J. Transmission risk beyond the village: entomological and human factors contributing to residual malaria transmission in an area approaching malaria elimination on the Thailand-Myanmar border. Malar j. 2019;18:221.

68. Dondorp AM, Yeung S, White L, Nguon C, Day NP, Socheat D, et al. Artemisinin resistance: current status and scenarios for containment. Nat Rev Microbiol. 2010;8:272.

69. Hii J, Rueda LM. Malaria vectors in the Greater Mekong Subregion: overview of malaria vectors and remaining challenges. SE Asian J Trop Med. 2013;44:73-165.

70. Gryseels C, Durnez L, Gerrets R, Uk S, Suon S, Set S, et al. Re-imagining malaria: heterogeneity of human and mosquito behaviour in relation to residual malaria transmission in Cambodia. Malar $\mathrm{J}$. 2015;14:165.

71. Hosking A. Efficacy of insecticide treated nets in South East Asia. In: Meek S, Kaviratne M, editors Malaria Consortium annotated bibliography; 2010. p.1-44.

72. Kolaczinski J, Macdonald M, Meek S. Vector control to eliminate artemisinin resistant malaria in the Greater Mekong Subregion. Lancet Infect Dis. 2014;14:9-11.

73. Dutta P, Khan SA, Khan AM, Borah J, Sarmah CK, Mahanta J. The effect of insecticide-treated mosquito nets (ITMNs) on Japanese encephalitis virus seroconversion in pigs and humans. Am J Trop Med Hyg. 2011;84:466-72.

74. Mondal D, Huda MM, Karmoker MK, Ghosh D, Matlashewski G.,Nabi SG, et al. Reducing visceral leishmaniasis by insecticide impregnation of bed-nets, Bangladesh. Emerg Infect Dis. 2013;19:11314.

75. Killeen GF, et al. Preventing childhood malaria in Africa by protecting adults from mosquitoes with insecticide-treated nets. PLoS Med. 2007;4:e229.

76. Google. (n.d.). [Tha Song Yang district, Tak province, Thailand]. Available online: https://is.gd/8Vutps. Access 5 May 2020.

77. NordNordWest. Locator map of Tak Province, Thailand. Available online: https://tl.wikipedia.org/wiki/Talaksan:Thailand_Tak_locator_map.svg. Access 5 May 2020.

\section{Figures}




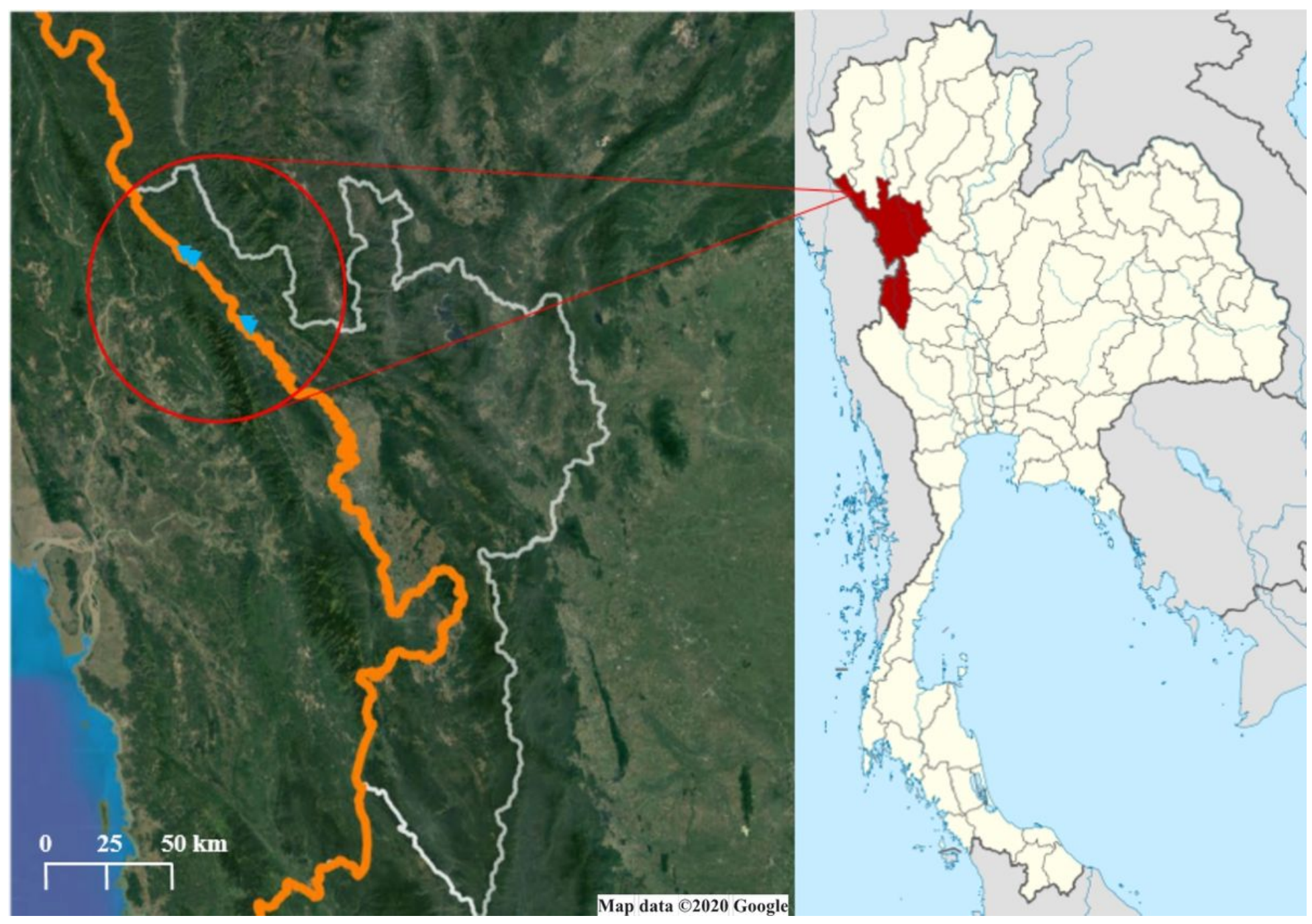

\section{Figure 1}

The study site: Tha Song Yang district, Tak province, Thailand [76] [77]. Note: The designations employed and the presentation of the material on this map do not imply the expression of any opinion whatsoever on the part of Research Square concerning the legal status of any country, territory, city or area or of its authorities, or concerning the delimitation of its frontiers or boundaries. This map has been provided by the authors.

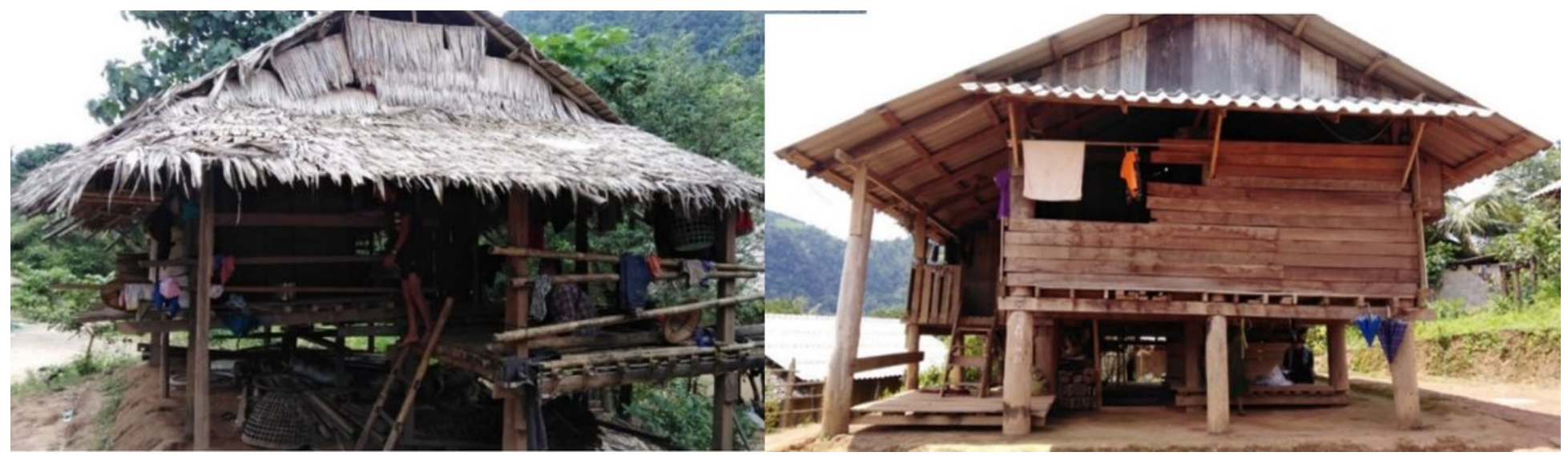

Figure 2 
Housing characteristics of the border population in Tha Song Yang district, Tak province, Thailand 